\title{
A Finite Element Method for Solving the Two Phase Stefan Problem in One Space Dimension ${ }^{1)}$
}

\author{
By
}

\author{
Masatake MORI*
}

\begin{abstract}
A finite element method based on time dependent basis functions is presented for solving a two phase Stefan problem for the heat equation in one space dimension. The stability and the convergence of the method are studied, and a numerical example is given.
\end{abstract}

\section{$\S 1$. A Two Phase Stefan Problem in One Space Dimension}

This paper is concerned with a finite element solution of the following two phase Stefan problem in one space dimension for the heat equation. Given the data $g_{1}(t), g_{2}(t), f_{1}(x), f_{2}(x)$ and $b$, find functions $u_{1}(x, t), u_{2}(x, t)$ and $x=s(t)$ in $0<x<L, 0<t \leqq T$, such that

$$
\begin{aligned}
& \left\{\begin{array}{l}
\frac{\partial u_{1}}{\partial t}=\sigma_{1} \frac{\partial^{2} u_{1}}{\partial x^{2}}, 0<x<s(t), 0<t \leqq T, \\
\frac{\partial u_{2}}{\partial t}=\sigma_{2} \frac{\partial^{2} u_{2}}{\partial x^{2}}, s(t)<x<L, 0<t \leqq T,
\end{array}\right. \\
& \left\{\begin{array}{l}
u_{1}(0, t)=g_{1}(t), u_{1}(s(t), t)=0,0<t \leqq T, \\
u_{2}(L, t)=g_{2}(t), u_{2}(s(t), t)=0,0<t \leqq T,
\end{array}\right. \\
& s(())=b,(), b \quad L, \\
& \left\{\begin{array}{l}
u_{1}(. t,()) \cdots f_{1}(r),(0)-x_{-}-t, \\
u_{2}(x, 0)=f_{2}(x), b \leqq x \leqq L,
\end{array}\right.
\end{aligned}
$$

Received November 11, 1976.

* Research Institute for Mathematical Sciences, Kyoto University, Kyoto 606, Japan.

1) Part of this work was reported in the Japan-France Seminar on Numerical Analysis and Functional Analysis, Tokyo and Kyoto, 1976, September [9]. 


$$
\frac{d s}{d t}=-\kappa_{1} \frac{\partial u_{1}}{\partial x}(s(t), t)+\kappa_{2} \frac{\partial u_{2}}{\partial x}(s(t), t), 0<t \leqq T,
$$

where $\sigma_{1}, \sigma_{2}, \kappa_{1}, \kappa_{2}$ and $L$ are positive constants, and $T$ is an arbitrarily fixed positive number. The last equation (1.5) expresses the heat balance and is called the Stefan condition. This condition (1.5) gives the speed of propagation of the free boundary. Specifically, the functions $u_{1}$ and $u_{2}$ may be interpreted as the temperature of the water existing in $0<x<s(t)$ and that of the ice existing in $s(t)<x<L$, respectively, which contact at the front $x=s(t)$ with each other.

For the moment we assume an appropriate smoothness for $g_{1}, g_{2}, f_{1}$ and $f_{2}$, and we make the following four assumptions for these Stefan data. First we assume that the initial data are bounded by quadratic functions both from above and from below:

Assumption A. There exist positive constants $A_{1}{ }^{\prime}, A_{2}{ }^{\prime}, B_{1}{ }^{\prime}$ and $B_{2}{ }^{\prime}$ such that

$$
\left\{\begin{array}{l}
B_{1}^{\prime}\left\{\left(\frac{x}{b}-2\right)^{2}-1\right\} \leqq f_{1}(x) \leqq A_{1}^{\prime}\left\{1-\frac{x^{2}}{b^{2}}\right\}, 0 \leqq x \leqq b \\
-A_{2}^{\prime}\left\{1-\frac{(L-x)^{2}}{(L-b)^{2}}\right\} \leqq f_{2}(x) \leqq-B_{2}^{\prime}\left\{\left(\frac{L-x}{L-b}-2\right)^{2}-1\right\} \\
\quad b \leqq x \leqq L
\end{array}\right.
$$

For the boundary data we make

Assumption B. There exist positive constants $A_{1}^{\prime \prime}, B_{1}^{\prime \prime}, A_{2}^{\prime \prime}$ and $B_{2}^{\prime \prime}$ such that

$$
\left\{\begin{array}{l}
0<3 B_{1}^{\prime \prime} \leqq g_{1}(t) \leqq A_{1}^{\prime \prime}, \quad 0 \leqq t \leqq T \\
-A_{2}{ }^{\prime \prime} \leqq g_{2}(t) \leqq-3 B_{2}{ }^{\prime \prime}<0, \quad 0 \leqq t \leqq T
\end{array}\right.
$$

Set

$$
\begin{cases}A_{1}=\max \left(A_{1}{ }^{\prime}, A_{1}{ }^{\prime \prime}\right), & B_{1}=\min \left(B_{1}{ }^{\prime}, B_{1}{ }^{\prime \prime}\right), \\ A_{2}=\max \left({A_{2}}^{\prime}, A_{2}{ }^{\prime \prime}\right), & B_{2}=\min \left({B_{2}}^{\prime}, B_{2}{ }^{\prime \prime}\right),\end{cases}
$$

and define 


$$
b_{m}=\frac{\kappa_{1} B_{1}}{\kappa_{2} A_{2}+\kappa_{1} B_{1}} L, \quad b_{M}=\frac{\kappa_{1} A_{1}}{\kappa_{1} A_{1}+\kappa_{2} B_{2}} L .
$$

We shall show later that $b_{m}$ and $b_{M}$ are a lower bound and an upper bound of $s(t)$, respectively. For the initial position $b$ of the free boundary we make

\section{Assumption C.}

$$
b_{m} \leqq b \leqq b_{M}
$$

Next we define

$$
\left\{\begin{array}{l}
\gamma_{+}=\frac{2}{L}\left(\kappa_{2} A_{2}+\kappa_{1} B_{1}\right)\left(\frac{A_{1}}{B_{1}}-\frac{B_{2}}{A_{2}}\right), \\
\gamma_{-}=\frac{2}{L}\left(\kappa_{1} A_{1}+\kappa_{2} B_{2}\right)\left(\frac{A_{2}}{B_{2}}-\frac{B_{1}}{A_{1}}\right),
\end{array}\right.
$$

and set

$$
\gamma=\max \left(\gamma_{+}, \gamma_{-}\right)
$$

and

$$
\eta=\max \left(\frac{b_{M}}{\sigma_{1}}, \frac{L-b_{m}}{\sigma_{2}}\right)
$$

Then finally we make

\section{Assumption D.}

$$
\gamma \eta \leqq 1
$$

From Assumptions $\mathrm{A}$ and $\mathrm{B}$ it is obvious that

$$
3 B_{1} \leqq A_{1}, \quad 3 B_{2} \leqq A_{2},
$$

so that from (1.11) we have $\gamma_{+}>0$ and $\gamma_{-}>0$. As we will see later the physical meaning of $\gamma_{+}$and $\gamma_{-}$would be more evident if we rewrite (1.11) equivalently as

(1. 16) $\quad \gamma_{+}=\frac{2 \kappa_{1} A_{1}}{b_{m}}-\frac{2 \kappa_{2} B_{2}}{L-b_{m}}, \quad \gamma_{-}=\frac{2 \kappa_{2} A_{2}}{L-b_{M}}-\frac{2 \kappa_{1} B_{1}}{b_{M}}$ 
In the preceding paper [7] we proposed a finite element scheme for solving the one phase problem and discussed the stability and the convergence of the scheme assuming that the initial data is bounded by a linear function from above. In the one phase problem the free boundary function $s(t)$ is monotone with respect to $t$, while in the two phase problem $s(t)$ is not monotone in general, so that we need quadratic or some other kind of functions as the bounding functions of the initial data in order that the maximum principle holds with our scheme. Cannon and Primicerio $[2,3]$ proved the existence and the uniqueness of the solution of (1.1)-(1.5) assuming that the initial data are bounded by exponential functions under a similar assumption as Assumption D.

Various numerical methods have been presented for solving the one phase Stefan problem in one space dimension. See, for example, Douglas and Gallie [4], Meyer [6], Bonnerot and Jamet [1], Nogi [10], Kawarada and Natori [5] and Mori [7]. See also Mori [8] for the numerical solution of the Stefan problem in two space dimension.

The purpose of the present paper is to present a finite element method for solving (1. 1)-(1.5), and to discuss the stability and the convergence of the method.

Before proceeding to the numerical method, we shall give here some remarks on the quantities $b_{m}, b_{M}, \gamma_{+}$and $\gamma_{\text {-. First we claim that under }}$ Assumptions A, B, C and D the solutions $u_{1}$ and $u_{2}$ of (1.1)(1.5) are always bounded by

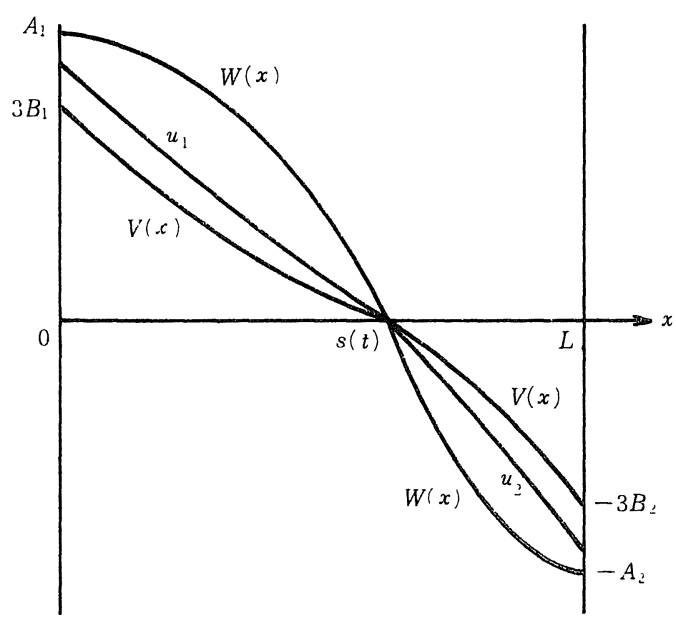

Fig. 1. quadratic functions as follows (see Fig. 1) provided that $|d s / d t| \leqq \gamma$ :

$$
\left\{\begin{array}{l}
V(x) \leqq u_{1}(x, t) \leqq W(x), \quad 0 \leqq x \leqq(t), 0 \leqq t \leqq T \\
W(x) \leqq u_{2}(x, t) \leqq V(x), \quad s(t) \leqq x \leqq L, \quad 0 \leqq t \leqq T
\end{array}\right.
$$

where 
(1. 18)

$$
\begin{aligned}
& W(x)=\left\{\begin{array}{l}
A_{1}\left\{1-\frac{x^{2}}{s^{2}(t)}\right\}, 0 \leqq x \leqq s(t) \\
-A_{2}\left\{1-\frac{(L-x)^{2}}{(L-s(t))^{2}}\right\}, s(t) \leqq x \leqq L
\end{array}\right. \\
& V(x)=\left\{\begin{array}{c}
B_{1}\left\{\left(\frac{x}{s(t)}-2\right)^{2}-1\right\}, 0 \leqq x \leqq s(t) \\
-B_{2}\left\{\left(\frac{L-x}{L-s(t)}-2\right)^{2}-1\right\}, s(t) \leqq x \leqq L .
\end{array}\right.
\end{aligned}
$$

The mathematical tool which can be used in order to prove these inequalities is the maximum principle. We shall show here only the inequality

$$
W(x)=A_{1}\left\{1-\frac{x^{2}}{s^{2}(t)}\right\} \geqq u_{1}(x, t), 0 \leqq x \leqq s(t)
$$

Define

$$
d_{1}(x, t) \equiv A_{1}\left\{1-\frac{x^{2}}{s^{2}(t)}\right\}-u_{1}(x, t),
$$

then, if $|d s / d t| \leqq \gamma$, we have

$$
\begin{gathered}
\left(\frac{\partial}{\partial t}-\sigma_{1} \frac{\partial^{2}}{\partial x^{2}}\right) d_{1}(x, t)=-A_{1}\left(\frac{\partial}{\partial t}-\sigma_{1} \frac{\partial^{2}}{\partial x^{2}}\right) \frac{x^{2}}{s^{2}(t)} \\
=\frac{2 A_{1} \sigma_{1}}{s^{2}(t)}\left(\frac{x^{2}}{\sigma_{1} s(t)} \times \frac{d s}{d t}+1\right) \\
\geqq \frac{2 A_{1} \sigma_{1}}{s^{2}(t)}\left(1-\frac{s(t)}{\sigma_{1}}\left|\frac{d s}{d t}\right|\right) \geqq \frac{2 A_{1} \sigma_{1}}{s^{2}(t)}(1-\eta \gamma)
\end{gathered}
$$

by (1.12) and (1.13), so that from Assumption D

$$
\left(\frac{\partial}{\partial t}-\sigma_{1} \frac{\partial^{2}}{\partial x^{2}}\right) d_{1}(x, t) \geqq 0
$$

Therefore, we obtain (1.20) using the maximum principle in view of Assumption A, the boundary condition (1.2) and Assumption B. The other three inequalities in (1.17) can be proved in a similar way.

If we assume that the derivatives of $u_{1}$ and $u_{2}$ at $x=s(t)$ exist (cf. $[2])$, then from (1.17) we have 


$$
\left\{\begin{array}{l}
-\frac{2 A_{1}}{s(t)} \leqq \frac{\partial u_{1}}{\partial x}(s(t), t) \leqq-\frac{2 B_{1}}{s(t)} \\
-\frac{2 A_{2}}{L-s(t)} \leqq \frac{\partial u_{2}}{\partial x}(s(t), t) \leqq-\frac{2 B_{2}}{L-s(t)},
\end{array}\right.
$$

so that from (1.5)

$$
\frac{2 \kappa_{1} A_{1}}{s(t)}-\frac{2 \kappa_{2} B_{2}}{L-s(t)} \geqq \frac{d s}{d t} \geqq-\left(\frac{2 \kappa_{2} A_{2}}{L-s(t)}-\frac{2 \kappa_{1} B_{1}}{s(t)}\right) .
$$

The bounding function $2 \kappa_{1} A_{1} / s(t)-2 \kappa_{2} B_{2} /(L-s(t))$ which bounds $\frac{d s}{d t}$ from above is nothing but the difference between the gradient of $W(x)$ at $x=s(t)-0$ and that of $V(x)$ at $x=s(t)+0$, and it decreases as the free boundary moves to the right, i.e. as $s(t)$ increases. It vanishes when the free boundary reaches the point $s(t)=b_{M}$ defined by (1.9), and hence we see that the free boundary $s(t)$ can never go rightwards beyond the point $x=b_{M}$. Similarly $s(t)$ can never go leftwards beyond the point $x=b_{m}$ defined by (1.9). Hence we have

$$
b_{m} \leqq s(t) \leqq b_{M} .
$$

On the other hand, the bounding function $2 \kappa_{1} A_{1} / s(t)-2 \kappa_{2} B_{2} /(L-s(t))$ bounding $\frac{d s}{d t}$ from above attains its maximum value $\gamma_{+}$defined by (1.16) at $s(t)=b_{m}$, and the function $-\left\{2 \kappa_{2} A_{2} /(L-s(t))-2 \kappa_{1} B_{1} / s(t)\right\}$ bounding $\frac{d s}{d t}$ from below attains its minimum value $-r_{-}$defined by (1.16) at $s(t)=b_{M}$, so that, as long as we assume (1.17), we have

$$
-\gamma_{-} \leqq \frac{d s}{d t} \leqq \gamma_{+} \quad \text { or } \quad\left|\frac{d s}{d t}\right| \leqq \gamma
$$

In $\S 3$ we shall show in a more consistent way that our finite element solutions also satisfy inequalities similar to (1.17) and (1.27).

\section{$\S 2$. Application of the Finite Element Method}

In this section we shall give a finite element scheme for solving (1.1)-(1.5) approximately. We write the approximate boundary function as $s_{n}(t)$ in order to show explicitly that it is an approximation of $s(t)$. 
First we fix time $t>0$, and divide the whole domain $0 \leqq x \leqq L$ into two subdomains $D_{1}$ and $D_{2}$ separated by the free boundary $x=s_{n}(t)$ :

$$
\left\{\begin{array}{l}
D_{1}=\left\{x \mid 0 \leqq x \leqq s_{n}(t)\right\}, \\
D_{2}=\left\{x \mid s_{n}(t) \leqq x \leqq L\right\} .
\end{array}\right.
$$

Then we partition $D_{1}$ and $D_{2}$ into $n_{1}$ equal and $n_{2}$ equal subintervals, respectively, and hence the free boundary $x=s_{n}(t)$ always coincides with a mesh point. The numbers of partition $n_{1}$ and $n_{2}$ are fixed throughout computation. Although each of $D_{1}$ and $D_{2}$ might be partitioned using a non-uniform mesh, we employed the equi-distant partition for simplicity. We denote each mesh point as $x_{j}$.

$$
\left\{\begin{aligned}
& x_{j}=j h_{1}(t), j=0,1, \cdots, n_{1} ; h_{1}(t)=\frac{1}{n_{1}} s_{n}(t), \\
& x_{j}=s_{n}(t)+\left(j-n_{1}\right) h_{2}(t), j=n_{1}, \cdots, n_{1}+n_{2} ; \\
& h_{2}(t)=\frac{1}{n_{2}}\left(L-s_{n}(t)\right) .
\end{aligned}\right.
$$

In the usual finite element method the mesh points are always fixed as $t$ varies, while in the present problem they change with time $t$.

We construct piecewise linear basis functions $\left\{\phi_{j}(x, t)\right\}$ as shown in Fig. 2:

$$
\phi_{j}(x, t)= \begin{cases}\frac{x-x_{j-1}}{x_{j}-x_{j-1}} ; & x_{j-1}<x \leqq x_{j} \\ \frac{x_{j+1}-x}{x_{j+1}-x_{j}} ; & x_{j}<x \leqq x_{j+1} \\ 0 ; & \text { otherwise. }\end{cases}
$$

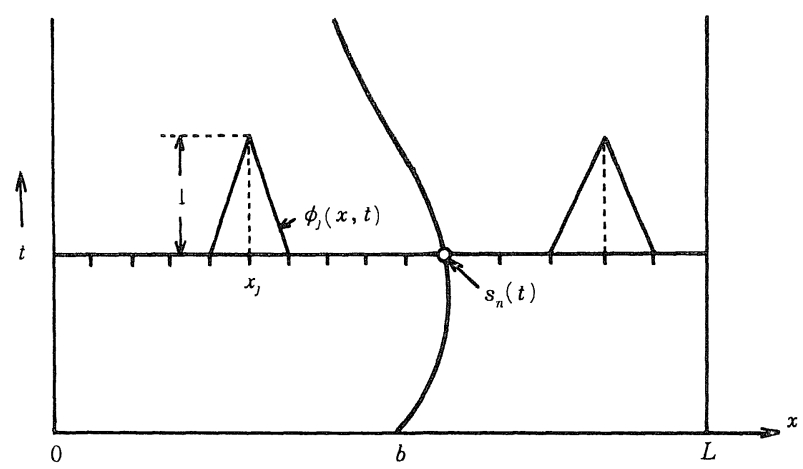

Fig. 2. 
For $\phi_{0}$ and $\phi_{n_{1}+n_{2}}$ we employ the components of (2.3) in $0 \leqq x \leqq x_{1}$ and $x_{n_{1}+n_{2}-1}<x \leqq L$, respectively. Note that the basis function $\phi_{j}(x, t)$ is time-dependent because $x_{j}$ depends on time $t$. See [7] for the derivatives of $\phi_{j}(x, t)$ with respect to $x$ and $t$.

Now we apply the Galerkin method based on the basis functions constructed above. We expand the approximate solutions $\widetilde{u}_{1}$ and $\widetilde{u}_{2}$ of $(1.1)-(1.5)$ in terms of linear combinations of $\phi_{j}(x, t)$ 's :

$$
\left\{\begin{array}{l}
\widetilde{u}_{1}(x, t)=\sum_{j=0}^{n_{1}} a_{j}(t) \phi_{j}(x, t) \\
\widetilde{u}_{2}(x, t)=\sum_{j=n_{1}}^{n_{1}+n_{2}} a_{j}(t) \phi_{j}(x, t),
\end{array}\right.
$$

where from (1.2)

$$
\left\{\begin{array}{l}
a_{0}(t)=g_{1}(t), \quad a_{n_{1}+n_{2}}(t)=g_{2}(t) \\
a_{n_{1}}(t)=0 .
\end{array}\right.
$$

If we substitute (2.4) into (1.1), multiply $\phi_{i}(x, t), i=1,2, \cdots, n_{1}-1$, $n_{1}+1, \cdots, n_{1}+n_{2}-1$, and integrate over $0 \leqq x \leqq L$, then we have the following system of ordinary differential equations:

$$
\left\{\begin{array}{l}
M_{1} \frac{d \boldsymbol{a}_{1}}{d t}=-\left(\sigma_{1} K_{1}+N_{1}\right) \boldsymbol{a}_{1} \\
M_{2} \frac{d \boldsymbol{a}_{2}}{d t}=-\left(\sigma_{2} K_{2}+N_{2}\right) \boldsymbol{a}_{2}
\end{array}\right.
$$

where

$$
\boldsymbol{a}_{1}=\left(\begin{array}{c}
a_{0} \\
a_{1} \\
\vdots \\
a_{n_{1}}
\end{array}\right), \quad \boldsymbol{a}_{2}=\left(\begin{array}{c}
a_{n_{1}} \\
a_{n_{1}+1} \\
\vdots \\
a_{n_{1}+n_{2}}
\end{array}\right)
$$

and $M_{1}, M_{2}$ are mass matrices, $K_{1}, K_{2}$ are stiffness matrices, and $N_{1}, N_{2}$ are velocity matrices [7]. We note that the matrices $M_{1}, K_{1}, N_{1}$ are of $\left(n_{1}-1\right) \times\left(n_{1}+1\right)$ and $M_{2}, K_{2}, N_{2}$ are of $\left(n_{2}-1\right) \times\left(n_{2}+1\right)$.

If we use the basis functions

$$
\psi_{j}(x, t)=\left\{\begin{array}{l}
1 ; \frac{1}{2}\left(x_{j-1}+x_{j}\right)<x \leqq \frac{1}{2}\left(x_{j}+x_{j+1}\right), \\
0 ; \text { otherwise }
\end{array}\right.
$$


instead of $\phi_{j}(x, t)$ in the computation of the mass matrices, we have equations for the lumped mass system, while the equations obtained using $\phi_{j}(x, t)$ 's are for the consistent mass system.

The explicit forms of $M_{\nu}, K_{\nu}, N_{\nu}(\nu=1,2)$ are as follows:

\section{Lumped Mass System:}

$$
\left(M_{\nu}\right)_{i j}=\left\{\begin{array}{l}
h_{\nu} ; j=i, \nu=1,2, \\
0 ; \text { otherwise }
\end{array}\right.
$$

$$
\left(K_{\nu}\right)_{i j}=\left\{\begin{array}{l}
-\frac{1}{h_{\nu}} ; j=i \pm 1, \nu=1,2, \\
\frac{2}{h_{\nu}} ; j=i, \nu=1,2, \\
0 ; \text { otherwise, }
\end{array}\right.
$$$$
\left(N_{1}\right)_{i j}=\left\{\begin{array}{l}
\mp \frac{1}{6 n_{1}}(3 i \pm 1) \frac{d s_{n}}{d t} ; j=i \pm 1, \\
\frac{1}{3 n_{1}} \times \frac{d s_{n}}{d t} ; j=i, \\
0 ; \text { otherwise, }
\end{array}\right.
$$

$$
\left(N_{2}\right)_{i j}=\left\{\begin{array}{l}
\mp \frac{1}{6 n_{2}}\left\{3\left(n_{1}+n_{2}-i\right) \mp 1\right\} \frac{d s_{n}}{d t} ; j=i \pm 1, \\
-\frac{1}{3 n_{2}} \times \frac{d s_{n}}{d t} ; j=i, \\
0 ; \text { otherwise. }
\end{array}\right.
$$

\section{Consistent Mass System:}

We have only to replace the mass matrices by the following ones:

$$
\left(M_{\nu}\right)_{i j}=\left\{\begin{array}{l}
\frac{1}{6} h_{\nu} ; j=i \pm 1, \nu=1,2, \\
\frac{2}{3} h_{\nu} ; j=i, \nu=1,2, \\
0 ; \text { otherwise. }
\end{array}\right.
$$

In the next step we discretize the time $t$ using an equal time mesh $\Delta t:$ 


$$
t=k \Delta t, k=0,1, \cdots, m ; t=\frac{T}{m},
$$

and replace the time derivatives of $\boldsymbol{a}_{1}$ and $\boldsymbol{a}_{2}$ by the time differences:

$$
\frac{d \boldsymbol{a}_{\nu}}{d t} \fallingdotseq \frac{\boldsymbol{a}_{\nu}(k \Delta t)-\boldsymbol{a}_{\nu}((k-1) \Delta t)}{\Delta t}, \nu=1,2 \text {. }
$$

Then we have simultaneous algebraic linear equations from (2.6). Similarly we make an approximation

$$
\frac{d s_{n}}{d t} \fallingdotseq \frac{\Delta s_{n}}{\Delta t}
$$

where $\Delta s_{n}$ is the increment of $s_{n}(t)$ from $t=(k-1) \Delta t$ to $t=k \Delta t$. We compute $\Delta s_{n}$ by replacing the gradients of $u_{1}$ and $u_{2}$ at $x=s(t)$ by those of $\widetilde{u}_{1}$ and $\widehat{u}_{2}$ at $x=s_{n}(t)$ in the right hand side of (1.5). Although the functions $\left\{\widetilde{u}_{1}, \widetilde{u}_{2}, s_{n}(t)\right\}$ should be computed simultanously and consistently, we employ an approximation such that we compute $\left\{\widetilde{u}_{1}, \widetilde{u}_{2}\right\}$ and $s_{n}(t)$ alternatively.

We summarize here the whole procedure obtained. We introduce a parameter $\theta$ with $0 \leqq \theta \leqq 1$, which denotes the mixing ratio of the forward difference $(\theta=0)$ and the backward one $(\theta=1)$ in the discretization of the time derivative.

\section{Initial Routine:}

$$
\left\{\begin{aligned}
a_{j}(0)=f_{1}\left(x_{j}\right) ; j & =0,1, \cdots, n_{1} ; \\
a_{j}(0)=f_{2}\left(x_{j}\right) ; j & =n_{1}+1, \cdots, n_{1}+n_{2}, \\
s_{n}(0) & =b .
\end{aligned}\right.
$$

\section{General Routine:}

Repeat the following process for $k=1,2, \cdots, m$.

(i) Compute $\Delta s_{n}(k \Delta t)$ and $s_{n}(k \Delta t)$ using $\boldsymbol{a}_{\nu}((k-1) \Delta t)$ and $s_{n}((k-1) \Delta t)$ by means of

$$
\begin{gathered}
\Delta s_{n}(k \Delta t)=\left\{\frac{\kappa_{1} n_{1} a_{n_{1}-1}((k-1) \Delta t)}{s_{n}((k-1) \Delta t)}+\frac{\kappa_{2} n_{2} a_{n_{1}+1}((k-1) \Delta t)}{L-s_{n}((k-1) \Delta t)}\right\} \Delta t, \\
s_{n}(k \Delta t)=s_{n}((k-1) \Delta t)+\Delta s_{n}(k \Delta t) .
\end{gathered}
$$


(ii) Compute $M_{\nu}, K_{\nu}, N_{\nu}, \nu=1,2$ using $\Delta s_{n}(k \Delta t)$ and $s_{n}(k \Delta t)$.

(iii) Solve the following linear equations for $\boldsymbol{a}_{1}(k \Delta t)$ and $\boldsymbol{a}_{2}(k \Delta t)$ :

$$
\begin{aligned}
\left\{M_{\nu}\right. & \left.+\theta \Delta t\left(\sigma_{\nu} K_{\nu}+N_{\nu}\right)\right\} \boldsymbol{a}_{\nu}(k \Delta t) \\
& =\left\{M_{\nu}-(1-\theta) \Delta t\left(\sigma_{\nu} K_{\nu}+N_{\nu}\right)\right\} \boldsymbol{a}_{\nu}((k-1) \Delta t), \nu=1,2 .
\end{aligned}
$$

\section{§. Stability}

In this section we consider the stability of the scheme (2.17)(2.21) obtained in $\S 2$. Here we shall confine ourselves to the case of the lumped mass system, while the stability of the scheme of the consistent mass system will be referred to at the end of this section.

In order to simplify the description we introduce

$$
\lambda_{1}(k \Delta t) \equiv \frac{\sigma_{1} n_{1}^{2} \Delta t}{s_{n}^{2}(k \Delta t)}, \quad \lambda_{2}(k \Delta t) \equiv \frac{\sigma_{2} n_{2}^{2} \Delta t}{\left(L-s_{n}(k \Delta t)\right)^{2}}
$$

and

$$
\beta_{1}(k \Delta t) \equiv \frac{\Delta s_{n}(k \Delta t)}{2 s_{n}(k \Delta t)}, \beta_{2}(k \Delta t) \equiv \frac{\Delta s_{n}(k \Delta t)}{2\left(L-s_{n}(k \Delta t)\right)}
$$

When the argument is $k \Delta t$, it may be omitted. $\lambda_{1}$ and $\lambda_{2}$ correspond to the parameter $\lambda=\sigma \Delta t / h$, where $h$ is the space mesh size, appearing in the finite difference or in the finite element method for the usual heat equation. In the present case, since $h_{1}$ and $h_{2}$ are time dependent, $\lambda_{1}$ and $\lambda_{2}$ also depend on $t$.

We define here two linear discrete operators $P_{1}$ and $P_{2}$ :

$$
\begin{aligned}
& P_{1}(k, j) w_{k}^{j} \equiv-\theta\left\{\lambda_{1}-\left(j-\frac{1}{3}\right) \beta_{1}\right\} w_{j-1}^{k}+\left\{1+2 \theta\left(\lambda_{1}+\frac{1}{3} \beta_{1}\right)\right\} w_{j}^{k} \\
&-\theta\left\{\lambda_{1}+\left(j+\frac{1}{3}\right) \beta_{1}\right\} w_{j+1}^{k}-(1-\theta)\left\{\lambda_{1}-\left(j-\frac{1}{3}\right) \beta_{1}\right\} w_{j-1}^{k-1} \\
&-\left\{1-2(1-\theta)\left(\lambda_{1}+\frac{1}{3} \beta_{1}\right)\right\} w_{j}^{k-1} \\
&-(1-\theta)\left\{\lambda_{1}+\left(j+\frac{1}{3}\right) \beta_{1}\right\} w_{j+1}^{k-1} \\
&: j=1,2, \cdots, n_{1}-1 ; k=1,2, \cdots, m,
\end{aligned}
$$


(3. 4) $P_{2}(k, j) w_{j}^{k} \equiv-\theta\left\{\lambda_{2}-\left(n_{1}+n_{2}-j+\frac{1}{3}\right) \beta_{2}\right\} w_{j-1}^{k}$

$$
\begin{aligned}
& +\left\{1+2 \theta\left(\lambda_{2}-\frac{1}{3} \beta_{2}\right)\right\} w_{j}^{k} \\
& -\theta\left\{\lambda_{2}+\left(n_{1}+n_{2}-j-\frac{1}{3}\right) \beta_{2}\right\} w_{j+1}^{k} \\
& -(1-\theta)\left\{\lambda_{2}-\left(n_{1}+n_{2}-j+\frac{1}{3}\right) \beta_{2}\right\} w_{j-1}^{k-1} \\
& -\left\{1-2(1-\theta)\left(\lambda_{2}-\frac{1}{3} \beta_{2}\right)\right\} w_{j}^{k-1} \\
& -(1-\theta)\left\{\lambda_{2}+\left(n_{1}+n_{2}-j-\frac{1}{3}\right) \beta_{2}\right\} w_{j+1}^{k-1} \\
& \quad: j=n_{1}+1, \cdots, n_{1}+n_{2}-1 ; k=1,2, \cdots, m .
\end{aligned}
$$

If we write

$$
a_{j}^{k}=a_{j}(k \Delta t)
$$

then the scheme (2.21) is expressed simply as

$$
\left\{\begin{array}{l}
P_{1}(k, j) a_{j}^{k}=0, j=1,2, \cdots, n_{1}-1, k=1,2, \cdots, m, \\
P_{2}(k, j) a_{j}^{k}=0, j=n_{1}+1, \cdots, n_{1}+n_{2}-1, k=1,2, \cdots, m .
\end{array}\right.
$$

In addition to the assumptions for the Stefan data given in $\S 1$, we make two assumptions for the choice of the parameters $n_{1}, n_{2}$ and $\Delta t$. We set

$$
\left\{\begin{array}{l}
K_{1} \equiv \max \left(\kappa_{1} A_{1}+\kappa_{2} B_{2}, \kappa_{2} A_{2}+\kappa_{1} B_{1}\right) \\
K_{2} \equiv \min \left(A_{1} B_{2}, A_{2} B_{1}\right)
\end{array}\right.
$$

\section{Assumption E.}

$$
\frac{K_{1}{ }^{3}}{\kappa_{1} \kappa_{2} K_{2}} \Delta t \leqq L^{2}
$$

Next writing

$$
\lambda_{1}^{M} \equiv \frac{\sigma_{1} n_{1}^{2} \Delta t}{b_{m}^{2}} \quad \text { and } \quad \lambda_{2}^{M} \equiv \frac{\sigma_{2} n_{2}{ }^{2} \Delta t}{\left(L-b_{M}\right)^{2}}
$$

we make 
Assumption $\mathbf{F}$ (lumped mass system).

$$
\frac{1}{2(1-\theta)} \geqq \max \left(\lambda_{1}^{M}\left(1+\frac{1}{6 n_{1}^{2}}\right), \lambda_{2}{ }^{M}\left(1+\frac{1}{6 n_{2}^{2}}\right)\right) .
$$

We shall see later that $b_{m} \leqq s_{n}(k \Delta t) \leqq b_{M}$, so that $\lambda_{1}{ }^{M}$ and $\lambda_{2}{ }^{M}$ are the upper bounds of $\lambda_{1}$ and $\lambda_{2}$, respectively.

Note that, Assumption $\mathrm{E}$ becomes trivial as $\Delta t \rightarrow 0$ and $n_{1}, n_{2} \rightarrow \infty$ while Assumption $\mathrm{F}$ remains essential except when $\theta=1$.

The aim of the present section is to establish the stability theorem, which will be obtained as a byproduct of the proof of the following finite element analogue of (1.17) (see Fig. 1):

$$
\left\{\begin{array}{c}
B_{1}\left\{\left(\frac{x_{j}}{s_{n}}-2\right)^{2}-1\right\} \leqq \widetilde{u}_{1}\left(x_{j}, k \Delta t\right) \leqq A_{1}\left\{1-\frac{x_{j}{ }^{2}}{s_{n}{ }^{2}}\right\} \\
j=0,1, \cdots n_{1}, \\
-A_{2}\left\{1-\frac{\left(L-x_{j}\right)^{2}}{\left(L-s_{n}\right)^{2}}\right\} \leqq \widetilde{u}_{2}\left(x_{j}, k \Delta t\right) \leqq-B_{2}\left\{\left(\frac{L-x_{j}}{L-s_{n}}-2\right)^{2}-1\right\} \\
j=n_{1}, \cdots, n_{1}+n_{2} .
\end{array}\right.
$$

We shall prove these inequalities by induction with respect to $k$, and for that purpose we need the following five lemmas.

Lemma 1. If

$$
\left\{\begin{array}{c}
2 B_{1}\left(1+\frac{1}{2 n_{1}}\right) \leqq n_{1} a_{n_{1}-1}^{l-1} \leqq 2 A_{1}\left(1-\frac{1}{2 n_{1}}\right), \\
2 B_{2}\left(1+\frac{1}{2 n_{2}}\right) \leqq-n_{2} a_{n_{1}+1}^{l-1} \leqq 2 A_{2}\left(1-\frac{1}{2 n_{2}}\right), \\
\left.b_{m} \leqq s_{n}((l-1)\rfloor t\right) \leqq b_{M l},
\end{array}\right.
$$

then

$$
-\gamma_{-}\left(1-\frac{1}{2 n_{2}}\right) \leqq \frac{\Delta s_{n}(l \Delta t)}{\Delta t} \leqq \gamma_{+}\left(1-\frac{1}{2 n_{1}}\right)
$$

Proof. Suppose we have $\Delta s_{n}(l \Delta t) \geqq 0$ when we compute the right hand side of (2.19). Then from (2.19), (3.12) and (3.13) we have 


$$
\begin{aligned}
\frac{\Delta s_{n}(l \Delta t)}{\Delta t} & \leqq \frac{2 \kappa_{1} A_{1}}{s_{n}((l-1) \Delta t)}\left(1-\frac{1}{2 n_{1}}\right)-\frac{2 \kappa_{2} B_{2}}{L-s_{n}((l-1) \Delta t)}\left(1+\frac{1}{2 n_{2}}\right) \\
& \leqq \frac{2 \kappa_{1} A_{1}}{b_{m}}\left(1-\frac{1}{2 n_{1}}\right)-\frac{2 \kappa_{2} B_{2}}{L-b_{m}}\left(1+\frac{1}{2 n_{2}}\right) \\
& \leqq\left(\frac{2 \kappa_{1} A_{1}}{b_{m}}-\frac{2 \kappa_{2} B_{2}}{L-b_{m}}\right)\left(1-\frac{1}{2 n_{1}}\right) \leqq \gamma_{+}\left(1-\frac{1}{2 n_{1}}\right) .
\end{aligned}
$$

We can prove the left inequality of (3.14) in the same way supposing that $\Delta s_{n}(l \Delta t) \leqq 0$.

Q.E.D.

Lemma 2. Under Assumption $E$,

$$
\begin{gathered}
\left\{\frac{2 \kappa_{2} A_{2}}{\left(L-b_{m}\right)^{2}}+\frac{2 \kappa_{1} B_{1}}{b_{m}^{2}}\right\} \Delta t \leqq 1, \quad\left\{\frac{2 \kappa_{1} A_{1}}{b_{M}^{2}}+\frac{2 \kappa_{2} B_{2}}{\left(L-b_{M}\right)^{2}}\right\} \Delta t \leqq 1, \\
b_{M}-b_{m} \geqq \gamma_{+} \Delta t, \quad b_{M}-b_{m} \geqq \gamma_{-} \Delta t .
\end{gathered}
$$

Proof. If we substitute the explicit forms of $b_{m}, b_{M}, \gamma_{-}$and $\gamma_{+}$into the above four inequalities, they are expressed equivalently as follows in that order:

$$
\begin{gathered}
\frac{2\left(\kappa_{2} A_{2}+\kappa_{1} B_{1}\right)^{3}}{\kappa_{1} \kappa_{2} A_{2} B_{1}} \Delta t \leqq L^{2}, \frac{2\left(\kappa_{1} A_{1}+\kappa_{2} B_{2}\right)^{3}}{\kappa_{1} \kappa_{2} A_{1} B_{2}} \Delta t \leqq L^{2}, \\
\left\{\begin{array}{l}
\frac{2\left(\kappa_{2} A_{2}+\kappa_{1} B_{1}\right)\left(\kappa_{1} A_{1}+\kappa_{2} B_{2}\right)^{2}}{\kappa_{1} \kappa_{2} A_{1} B_{2}} \Delta t \leqq L^{2}, \\
\frac{2\left(\kappa_{1} A_{1}+\kappa_{2} B_{2}\right)\left(\kappa_{2} A_{2}+\kappa_{1} B_{1}\right)^{2}}{\kappa_{1} \kappa_{2} A_{2} B_{1}} \Delta t \leqq L^{2} .
\end{array}\right.
\end{gathered}
$$

It is evident from Assumption $E$ that all these inequalities are valid.

Q.E.D.

Lemma 3. Under the same hypothesis of Lemma 1 and under Assumption E,

$$
b_{m} \leqq s_{n}(l \Delta t) \leqq b_{M} .
$$

Proof. Since

$$
-\gamma_{-} \Delta t \leqq \Delta s_{n}(l \Delta t) \leqq \gamma_{+} \Delta t
$$


from Lemma 1, the increment of $s_{n}$ never exceeds $\gamma_{+} \Delta t$ or $\gamma_{-} \Delta t$ at each step. When $\Delta s_{n}(l \Delta t) \geqq 0$, we suppose $s_{n}((l-1) \Delta t) \leqq b_{M}-\delta, 0 \leqq \delta \leqq \gamma_{+} \Delta t$ in view of (3.20) and prove that $s_{n}(l \Delta t) \leqq b_{M}$, while when $\Delta s_{n}(l \Delta t) \leqq 0$, we suppose $b_{m}+\delta \leqq s_{n}((l-1) \Delta t), 0 \leqq \delta \leqq \gamma_{-} \Delta t$ and prove that $b_{m} \leqq s_{n}(l \Delta t)$.

First assume that $\Delta s_{n}(l \Delta t) \geqq 0$. Suppose that

$$
s_{n}((l-1) \Delta t) \leqq b_{M}-\delta, 0 \leqq \delta \leqq \gamma_{+} \Delta t,
$$

where $b_{m} \leqq b_{M}-\delta$ because of (3.16). Define an auxiliary function

$$
F_{+}(\grave{\delta}) \equiv\left\{\frac{2 \kappa_{1} A_{1}}{b_{M}-\delta}-\frac{2 \kappa_{2} B_{2}}{L-b_{M}+\delta}\right\} \Delta t
$$

Then it is obvious from (2.19) in view of (3.21) and (3.12) that

$$
0 \leqq \Delta s_{n}(l \Delta t) \leqq F_{+}(\delta),
$$

so that, if $F_{+}(0) \leqq \delta$, then

(3.24) $\quad s_{n}(l \Delta t)=s_{n}((l-1) \Delta t)+\Delta s_{n}(l \Delta t) \leqq s_{n}((l-1) \Delta t)+F_{+}(\delta)$

$$
\leqq s_{n}((l-1) \Delta t)+\delta \leqq b_{M}
$$

from (3.21). So what we need is to show that $F_{+}(\delta) \leqq \delta$. It is easy to see that $F_{+}(\delta) \leqq \delta$ follows from

$$
\left.\frac{d}{d \delta} F_{+}(\delta)\right|_{\hat{\delta}=0} \leqq 1
$$

and

$$
F_{+}\left(\gamma_{+} \Delta t\right) \leqq \gamma_{+} \Delta t
$$

If we write (3.25) explicitly using (3.22), we have

$$
\left\{\frac{2 \kappa_{1} A_{1}}{b_{M}^{2}}+\frac{2 \kappa_{2} B_{2}}{\left(L-b_{M}\right)^{2}}\right\} \Delta t \leqq 1
$$

which is guaranteed by (3.15). The inequality $(3.26)$, on the other hand, can be shown to hold as follows using (3.16) and (1.16):

$$
\begin{aligned}
F_{+}\left(\gamma_{+} \Delta t\right) & =\left\{\frac{2 \kappa_{1} A_{1}}{b_{M}-\gamma_{+} \Delta t}-\frac{2 \kappa_{2} B_{2}}{L-b_{M}+\gamma_{+} \Delta t}\right\} \Delta t \\
& \leqq\left\{\frac{2 \kappa_{1} A_{1}}{b_{m}}-\frac{2 \kappa_{2} B_{2}}{L-b_{m}}\right\} \Delta t=\gamma_{+} \Delta t,
\end{aligned}
$$

which verifies $F_{+}(\delta) \leqq \delta$. 
When $\Delta s_{n}(l \Delta t) \leqq 0$, we define

$$
F_{-}(\delta) \equiv\left\{\frac{2 \kappa_{2} A_{2}}{L-b_{m}-\delta}-\frac{2 \kappa_{1} B_{1}}{b_{m}+\delta}\right\} \Delta t .
$$

Then $b_{m} \leqq s_{n}(l \Delta t)$ can be derived from $b_{m}+\delta \leqq s_{n}((l-1) \Delta t), 0 \leqq \delta \leqq \gamma_{-} \Delta t$ in the same way as given above.

Q.E.D.

Lemma A (lumpcd mass system). Under the same hypothesis of Lemma 1 and under Assumptions $D$ and $F$,

$$
\left\{\begin{array}{l}
\frac{1}{2(1-\theta)} \geqq \lambda_{1}(l \Delta t)+\frac{1}{3} \beta_{1}(l \Delta t) \geqq n_{1}\left|\beta_{1}(l \Delta t)\right|, \\
\frac{1}{2(1-\theta)} \geqq \lambda_{2}(l \Delta t)-\frac{1}{3} \beta_{2}(l \Delta t) \geqq n_{2}\left|\beta_{2}(l \Delta t)\right| .
\end{array}\right.
$$

Proof. We begin with the first inequality.

$$
\begin{aligned}
& \frac{1}{2(1-\theta)}-\lambda_{1}-\frac{1}{3} \beta_{1}=\frac{1}{2(1-\theta)}-\lambda_{1}-\frac{\sigma_{1} \Delta t}{6 s_{n}{ }^{2}} \times \frac{s_{n}}{\sigma_{1}} \times \frac{\Delta s_{n}}{\Delta t} \\
& \geqq \frac{1}{2(1-\theta)}-\lambda_{1}-\frac{\sigma_{1} \Delta t}{6 s_{n}{ }^{2}} \eta \gamma \\
& \geqq \frac{1}{2(1-\theta)}-\lambda_{1}\left(1+\frac{1}{6 n_{1}{ }^{2}}\right) \quad(\text { from Assumption D) } \\
& \left.\geqq \frac{1}{2(1-\theta)}-\lambda_{M}\left(1+\frac{1}{6 n_{1}{ }^{2}}\right) \geqq 0 \text { (from Assumption } \mathrm{F}\right) .
\end{aligned}
$$

The second inequality can be proved as follows.

$$
\begin{gathered}
\lambda_{1}+\frac{1}{3} \beta_{1}-n_{1}\left|\beta_{1}\right| \geqq \lambda_{1}-\left(n_{1}+\frac{1}{3}\right) \frac{\sigma_{1} \Delta t}{2 s_{n}{ }^{2}} \times \frac{s_{n}}{\sigma_{1}}\left|\frac{\Delta s_{n}}{\Delta t}\right| \\
\geqq \lambda_{1}\left\{1-\frac{\left(n_{1}+1 / 3\right)}{2 n_{1}{ }^{2}} \eta \gamma\right\} \geqq \lambda_{1}(1-\eta \gamma) \geqq 0 .
\end{gathered}
$$

The other inequalities can also be proved in a similar way. Q.E.D.

Finally using Lemma 4, we have the following local maximum principle for the present scheme, the proof of which is exactly the same as that of Lemma 1 in the preceding paper [7]. 
Lemma 5 (lumped mass system). If (3.30) holds for $l=k$ and if

$$
p_{j}^{k} \geqq 0, \quad j=1,2, \cdots, n_{1}-1, n_{1}+1, \cdots, n_{1}+n_{2}-1,
$$

then the following maximum principle holds for the scheme

$$
\left\{\begin{array}{l}
P_{1}(k, j) w_{j}^{k}=p_{j}^{k}, \quad j=1,2, \cdots, n_{1}-1, \\
P_{2}(k, j) w_{j}^{k}=p_{j}^{k}, \quad j=n_{1}+1, \cdots, n_{1}+n_{2}-1,
\end{array}\right.
$$

locally at $k$ :

(3. 35) $\min \left\{w_{0}^{k}, w_{n_{1}}^{k}, w_{\min , 1}^{k-1}\right\} \leqq w_{j}^{k} \leqq \max \left\{w_{0}^{k}, w_{n_{1}}^{k}, w_{\max , 1}^{k-1}\right\}+p_{j}^{k}$,

$$
j=0,1, \cdots, n_{1},
$$

(3. 36) $\min \left\{w_{n_{1}}^{k}, w_{n_{1}+n_{2}}^{k}, w_{\min , 2}^{k-1}\right\} \leqq w_{j}^{k} \leqq \max \left\{w_{n_{1}}^{k}, w_{n_{1}+n_{2}}^{k}, w_{\max , 2}^{k-1}\right\}+p_{j}^{k}$,

$$
j=n_{1}, n_{1}+1, \cdots, n_{1}+n_{2},
$$

where

$$
\left\{\begin{array}{l}
w_{\min , 1}^{k-1}=\min _{0 \leqq j \leqq n_{1}} w_{j}^{k-1}, \quad w_{\max , 1}^{k-1}=\max _{0 \leqq j \leqq n_{1}} w_{j}^{k-1}, \\
w_{\min , 2}^{k-1}=\min _{n_{1} \leqq j \leqq n_{1}+n_{2}} w_{j}^{k-1}, w_{\max , 2}^{k-1}=\max _{n_{1} \leqq j \leqq n_{1} \dagger n_{2}} w_{j}^{k-1} .
\end{array}\right.
$$

Now we are ready to verify the inequalities (3.11). We introduce the following quantities for simplicity.

$$
\begin{aligned}
& \widetilde{V}_{j} \equiv\left\{\begin{array}{l}
A_{1}\left\{1-\frac{j^{2}}{n_{1}^{2}}\right\}, j=0,1, \cdots, n_{1}, \\
-A_{2}\left\{1-\frac{\left(n_{1}+n_{2}-j\right)^{2}}{n_{2}^{2}}\right\}, j=n_{1}, \cdots, n_{1}+n_{2} .
\end{array}\right. \\
& \widetilde{V}_{J} \equiv\left\{\begin{array}{c}
B_{1}\left\{\left(\frac{j}{n_{1}}-2\right)^{2}-1\right\}, j=0,1, \cdots, n_{1}, \\
-B_{2}\left\{\left(\frac{n_{1}+n_{2}-j}{n_{2}}-2\right)^{2}-1\right\}, j=n_{1}, \cdots, n_{1}+n_{2} .
\end{array}\right.
\end{aligned}
$$

Lemma 6 (lumped mass system). Under Assumptions $A, B, C$, $D, E$ and $F$, the following inequalities hold for $k=0,1, \cdots, m$.

$$
\widetilde{V}_{j} \leqq a_{j}^{k} \leqq \widetilde{W}_{j}, j=0, \cdots, n_{1},
$$




$$
\widetilde{W}_{j} \leqq a_{j}^{k} \leqq \widetilde{V}_{j}, j=n_{1}, \cdots, n_{1}+n_{2},
$$

$$
b_{m} \leqq s_{n}(k \Delta t) \leqq b_{M}
$$

Proof. We shall prove this lemma by induction with respect to $k$. When $k=0$, (3.40) and (3.41) are nothing but (1.6) of Assumption $\mathrm{A}$, and (3.42) is (1.10) of Assumption C.

Suppose that (3.40), (3.41) and (3.42) hold for $k=l-1$. If we put $j=n_{1}-1$ in (3.40) and $j=n_{1}+1$ in (3.41), we have

$$
\begin{aligned}
& 2 B_{1}\left(1+\frac{1}{2 n_{1}}\right) \leqq n_{1} a_{n_{1}-1}^{l-1} \leqq 2 A_{1}\left(1-\frac{1}{2 n_{1}}\right), \\
& 2 B_{2}\left(1+\frac{1}{2 n_{2}}\right) \leqq-n_{2} a_{n_{1}+1}^{l-1} \leqq 2 A_{2}\left(1-\frac{1}{2 n_{2}}\right) .
\end{aligned}
$$

From these inequalities together with (3.42), we see that the hypothesis of Lemma 1 holds. Then, since the hypothesis of Lemma 3 also holds, we immediately see that $(3.42)$ is valid for $k=l$. Next we define the difference between $\widetilde{W}_{j}$ and $a_{j}^{l}$, i.e.

$$
d_{j}^{l} \equiv \widetilde{W}_{j}-a_{j}^{l},
$$

and prove that $d_{j}^{l} \geqq 0$, i.e. the second inequality in (3.40), by using Lemma 5. The hypothesis of Lemma 4 holds, so that (3.30) is valid. In order to use Lemma 5, we need to show that $P_{1}(l, j) d_{j}^{l}=p_{j}^{l} \geqq 0$, which corresponds to (3.33). The inequality $p_{j}^{l} \geqq 0$ can be proved as follows from Assumption D:

$$
\begin{aligned}
P_{1}(l, j) d_{j}^{l} & =P_{1}(l, j)\left(\widetilde{W}_{j}-a_{j}^{l}\right)=P_{1}(l, j) \widetilde{W}_{j}\left(=p_{j}^{l}\right) \\
& =-\left(\lambda_{1}+\frac{1}{3} \beta_{1}\right)\left(\widetilde{W}_{j-1}-2 \widetilde{W}_{j}+\widetilde{W}_{j+1}\right)+j \beta_{1}\left(\widetilde{W}_{j-1}-\widetilde{W}_{j+1}\right) \\
& =\frac{2 A_{1} \sigma_{1} \Delta t}{s_{n}^{2}}\left\{1+\frac{1}{n_{1}{ }^{2}}\left(j^{2}+\frac{1}{6}\right) \frac{s_{n} \Delta s_{n}}{\sigma_{1} \Delta t}\right\} \\
& \geqq \frac{2 A_{1} \sigma_{1} \Delta t}{s_{n}^{2}}\left\{1-\frac{s_{n}}{\sigma_{1}}\left|\frac{\Delta s_{n}}{\Delta t}\right|\right\} \geqq \frac{2 A_{1} \sigma_{1} \Delta t}{s_{n}^{2}}(1-\eta \gamma) \geqq 0, \\
& j=1,2, \cdots, n_{1}-1 .
\end{aligned}
$$

In view of the boundary condition 


$$
\widetilde{W}_{0} \geqq a_{0}^{l}, \quad \widetilde{W}_{n_{1}}=a_{n_{1}}^{k}=0
$$

from Assumption $\mathrm{B}$, we have $\widetilde{W}_{j} \geqq a_{j}^{l}$ by Lemma 5 .

The other three inequalities can also be verified in the same way with the aid of the following three inequalities:

$$
\left\{\begin{array}{l}
P_{1}(l, j)\left(a_{j}^{l}-\widetilde{V}_{j}\right) \geqq 0, j=1,2, \cdots, n_{1}-1, \\
P_{2}(l, j)\left(a_{j}^{l}-\widetilde{W}_{j}\right) \geqq 0, j=n_{1}+1, \cdots, n_{1}+n_{2}-1, \\
P_{2}(l, j)\left(\widetilde{V}_{j}-a_{j}^{l}\right) \geqq 0, j=n_{1}+1, \cdots, n_{1}+n_{2}-1 . \quad \text { Q.E.D. }
\end{array}\right.
$$

Lemma 6 asserts that, under Assumptions A, B, C, D, E and F, the maximum principle in the sense of Lemma 5 holds for the present scheme (3.6) locally at each $k=1,2, \cdots, m$, so that for stability we have

Theorem 1 (lumped mass system). Under Assumptions A, B, C, $D, E$ and $F$, the scheme

$$
\left\{\begin{array}{l}
P_{1}(k, j) a_{j}^{k}=0, j=1,2, \cdots, n_{1}-1 \\
P_{2}(k, j) a_{j}^{k}=0, j=n_{1}+1, \cdots, n_{1}+n_{2}-1,
\end{array}\right.
$$

is stable in the sense that the following maximum principle holds locally at $k=1,2, \cdots, m$ :

$$
\left\{\begin{array}{l}
0 \leqq a_{j}^{k} \leqq \max \left(a_{0}^{k}, a_{\max , 1}^{k-1}\right), j=1,2, \cdots, n_{1}-1 \\
\min \left(a_{n_{1} \vdash n_{2}}^{k}, a_{\min , 2}^{k-1}\right) \leqq a_{j}^{k} \leqq 0, j=n_{1}+1, \cdots, n_{1}+n_{2}-1 .
\end{array}\right.
$$

Theorem 1 can be shown to hold also for the scheme of the consistent mass system

$$
\begin{aligned}
{[1-6} & \left.\theta\left\{\lambda_{1}-\left(j-\frac{1}{3}\right) \beta_{1}\right\}\right] a_{j-1}^{k}+\left\{4+12 \theta\left(\lambda_{1}+\frac{1}{3} \beta_{1}\right)\right\} a_{j}^{k} \\
+ & {\left[1-6 \theta\left\{\lambda_{1}+\left(j+\frac{1}{3}\right) \beta_{1}\right\}\right] a_{j+1}^{k} } \\
= & {\left[1+6(1-\theta)\left\{\lambda_{1}-\left(j-\frac{1}{3}\right) \beta_{1}\right\}\right] a_{j-1}^{k-1} } \\
+ & \left\{4-12(1-\theta)\left(\lambda_{1}+\frac{1}{3} \beta_{1}\right)\right\} a_{j}^{k-1}
\end{aligned}
$$




$$
\begin{aligned}
& +\left[1+6(1-\theta)\left\{\lambda_{1}+\left(j+\frac{1}{3}\right) \beta_{1}\right\}\right] a_{j+1}^{k-1}, \\
& j=1,2, \cdots, n_{1}-1 ; k=1,2, \cdots, m, \\
& {\left[1-6 \theta\left\{\lambda_{2}-\left(n_{1}+n_{2}-j+\frac{1}{3}\right) \beta_{2}\right\}\right] a_{j-1}^{k}+\left\{4+12\left(\lambda_{2}-\frac{1}{3} \beta_{2}\right)\right\} a_{j}^{k}} \\
& +\left[1-6 \theta\left\{\lambda_{2}+\left(n_{1}+n_{2}-j-\frac{1}{3}\right) \beta_{2}\right\}\right] a_{j+1}^{k} \\
& =\left[1+6(1-\theta)\left\{\lambda_{2}-\left(n_{1}+n_{2}-j+\frac{1}{3}\right) \beta_{2}\right\}\right] a_{j-1}^{k-1} \\
& +\left\{4-12(1-\theta)\left(\lambda_{2}-\frac{1}{3} \beta_{2}\right)\right\} a_{j}^{k-1} \\
& +\left[1+6(1-\theta)\left\{\lambda_{2}+\left(n_{1}+n_{2}-j-\frac{1}{3}\right) \beta_{2}\right\}\right] a_{j+1}^{k-1}, \\
& \quad j=n_{1}+1, \cdots, n_{1}+n_{2}-1 ; k=1,2, \cdots, m
\end{aligned}
$$

if we replace Assumption $F$ by the following one. We define here

$$
\lambda_{1}^{m} \equiv \frac{\sigma_{1} n_{1}^{2} \Delta t}{b_{M}^{2}}, \lambda_{2}^{m} \equiv \frac{\sigma_{2} n_{2}^{2} \Delta t}{\left(L-b_{m}\right)^{2}} .
$$

Assumption F (consistent mass system).

$$
\begin{aligned}
& \frac{1}{3(1-\theta)} \geqq \lambda_{1}^{M}\left(1+\frac{1}{6 n_{1}^{2}}\right), \quad \lambda_{1}^{m}\left(1-\frac{1}{2 n_{1}}\right) \geqq \frac{1}{6 \theta}, \\
& \frac{1}{3(1-\theta)} \geqq \lambda_{2}^{M}\left(1+\frac{1}{6 n_{2}^{2}}\right), \quad \lambda_{2}^{m}\left(1-\frac{1}{2 n_{2}}\right) \geqq \frac{1}{6 \theta} .
\end{aligned}
$$

It is easy to see that for $k=0,1, \cdots, m$

$$
\left\{\begin{array}{l}
\lambda_{1}^{m} \leqq \lambda_{1}(k \Delta t) \leqq \lambda_{1}^{M} \\
\lambda_{2}^{m} \leqq \lambda_{2}(k \Delta t) \leqq \lambda_{2}^{M}
\end{array}\right.
$$

\section{$\S 4$. Convergence}

In this section we shall show that the approximate solution $\left\{\widetilde{u}_{1}, \widetilde{u}_{2}, s_{n}\right\}$ converges to the solution $\left\{u_{1}, u_{2}, s\right\}$ of (1.1)-(1.5) uniformly as $\Delta t \rightarrow 0$. 
For simplicity we shall confine ourselves to the case of the lumped mass system with $\theta=1$, i.e.

(4. 1)

$$
\left\{\begin{array}{c}
-\left\{\lambda_{1}-\left(j-\frac{1}{3}\right) \beta_{1}\right\} a_{j-1}^{k}+\left\{1+2\left(\lambda_{1}+\frac{1}{3} \beta_{1}\right)\right\} a_{j}^{k} \\
-\left\{\lambda_{1}+\left(j+\frac{1}{3}\right) \beta_{1}\right\} a_{j+1}^{k}=a_{j}^{k-1}, j=1,2, \cdots, n_{1}-1, \\
-\left\{\lambda_{2}-\left(n_{1}+n_{2}-j+\frac{1}{3}\right) \beta_{2}\right\} a_{j-1}^{k}+\left\{1+2\left(\lambda_{2}-\frac{1}{3} \beta_{2}\right)\right\} a_{j}^{k} \\
-\left\{\lambda_{2}+\left(n_{1}+n_{2}-j-\frac{1}{3}\right) \beta_{2}\right\} a_{j+1}^{k}=a_{j}^{k-1} \\
j=n_{1}+1, \cdots, n_{1}+n_{2}-1 .
\end{array}\right.
$$

Now we make two assumptions for the limit $\Delta \iota \rightarrow 0, n_{1}, n_{2} \rightarrow \infty$ and for the smoothness of the initial and the boundary data.

\section{Assumption G.}

$$
\left\{\begin{array}{l}
\lambda_{1}^{M}=\frac{\sigma_{1} n_{1}^{2} \Delta t}{b_{m}^{2}}=\text { constant } \\
\lambda_{2}^{M}=\frac{\sigma_{2} n_{2}^{2} \Delta t}{\left(L-b_{M}\right)^{2}}=\text { constant }
\end{array}\right.
$$

\section{Assumption $\mathrm{H}$.}

$$
\begin{gathered}
f_{1}, f_{2} \in C^{2}(x), g_{1}, g_{2} \in C^{1}(t) \\
\left\{\begin{array}{l}
g_{1}(0)=f_{1}(0), \frac{d g_{1}}{d t}(0)=\sigma_{1} \frac{d^{2} f_{1}}{d x^{2}}(0) \\
g_{2}(0)=f_{2}(0), \frac{d g_{2}}{d t}(0)=\sigma_{2} \frac{d^{2} f_{2}}{d x^{2}}(L)
\end{array}\right.
\end{gathered}
$$

We extend the approximate solutions $\left\{\widetilde{u}_{1}, \widetilde{u}_{2}, s_{n}(t)\right\}$ which are defined only at the discrete points $t=k \Delta t$ to those defined also at the intermediate values of $t$, i.e. $k \Delta t<t \leqq(k+1) \Delta t$, as follows. First we define $s_{n}(t)$ by means of the linear interpolation

$$
s_{n}(t)=s_{n}(k \Delta t)+\alpha \Delta s_{n}((k+1) \Delta t), k \Delta t<t \leqq(k+1) \Delta t,
$$


where

$$
\alpha=\frac{t-k \Delta t}{\Delta t}
$$

Accordingly, $\phi_{j}(x, t)$ is also defined for any $t$. Next we extend $a_{j}(t)$ again by means of the linear interpolation based on $a_{j}(k \Delta t)$ and $a_{j}((k+1) \Delta t)$. Once $a_{j}(t)$ is extended, then $\widetilde{u}_{1}(x, t)$ and $\widetilde{u}_{2}(x, t)$ are defined by (2.4). Note that the value of $\widetilde{u}_{1}(x, t)$ at $j h_{1}<x \leqq(j+1) h_{1}$ is equal to that of the linear interpolation based on $a_{j}(t)$ and $a_{j+1}(t)$.

In regard to the extended $\left\{s_{n}(t)\right\}$, we have

Lemma 7. The functions $\left\{s_{n}(t)\right\}$ form an equicontinuous uniformly bounded family in $0 \leqq t \leqq T$.

Proof. From Lemmas 6 and 3 , we have $b_{m} \leqq s_{n}(k \Delta t) \leqq b_{M}, k=0,1$, $\cdots, m$, so that by the definition (4.5) of $s_{n}(t)$ we see that $s_{n}(t)$ are uniformly bounded. Similarly from Lemmas 6 and 1, we have

$$
\left|\Delta s_{n}(k \Delta t)\right| \leqq \gamma \Delta t, \quad k=1,2, \cdots, m .
$$

This inequality together with (4.5) implies the equicontinuity of $\left\{s_{n}(t)\right\}$.

Q.E.D.

According to this lemma, we can extract a subsequence from $\left\{s_{n}(t)\right\}$ which converges. Namely, if we write this subsequence as $\left\{s_{n}(t)\right\}$ anew, and if we let the limit function be $s_{\infty}(t)$, then for any $\varepsilon>0$ we have

$$
\left|s_{n}(t)-s_{\infty}(t)\right|<\varepsilon
$$

for sufficiently large $n$.

In the next step we regard the boundary function $s(t)$ to be the given function $s_{\infty}(t)$, which is uniformly Lipschitz continuous, and let $\widehat{u}_{1}$ be the solution in $D_{1}$ and $\hat{u}_{2}$ be that in $D_{2}$ of the heat equation $(1.1)-(1.4)$ associated with the moving boundary $s_{\infty}(t)$. Then, if we consider the domains $D_{1}$ and $D_{2}$ separately, we can prove in the same way as in the proof of $\S 4$ of [7] that $\widetilde{u}_{1}$ and $\widetilde{u}_{2}$ converge uniformly to $\hat{u}_{1}$ and $\hat{u}_{2}$, respectively, as $\Delta t \rightarrow 0, n_{1}, n_{2} \rightarrow \infty$ under Assumptions A, B, C. $\mathrm{D}, \mathrm{E}, \mathrm{F}, \mathrm{G}$ and $\mathrm{H}$. The only different point is that in [7] $s_{n}(t)$ and $s_{\infty}(t)$ are monotone while in the present case they are not monotone, so 
that Lemma 4 in [7] does not hold. We see, however, that the conclusion of Lemma 4 of [7] is also valid with $A$ replaced by $2 A_{1} / b_{m}$ in $D_{1}$ and by $2 A_{2} /\left(L-b_{M}\right)$ in $D_{2}$ if we remind of (4.7) and the discussion about the inequalities (1.17) in $\S 1$.

What is left to be done is to show that the functions $\hat{u}_{1}, \hat{u}_{2}$ and $s_{\infty}(t)$ satisfy the Stefan condition (1.5). For that purpose we define the second difference of $a_{j}^{k}$ :

$$
\left\{\begin{array}{l}
c_{j}^{k}=\frac{a_{j-1}^{k}-2 a_{j}^{k}+a_{j+1}^{k}}{h_{1}^{2}}, j=1,2, \cdots, n_{1}-1, \\
c_{j}^{k}=\frac{a_{j-1}^{k}-2 a_{j}^{k}+a_{j+1}^{k}}{h_{2}^{2}}, j=n_{1}+1, \cdots, n_{1}+n_{2}-1 .
\end{array}\right.
$$

Then, by considering $D_{1}$ and $D_{2}$ separately, we can prove the following lemma. For the proof see Lemma 5 in [7].

Lemma 8 (lumped mass system, $\theta=1$ ). Under Assumption $A$, $B, C, D, E, F$ and $H$,

(4. 10) $\left|c_{j}^{k}\right| \leqq M, j=1,2, \cdots, n_{1}-1, n_{1}+1, \cdots, n_{1}+n_{2}-1 ; k=1,2, \cdots, m$.

We can also show that the following inequalities are valid as in the same way as in the proof of Lemma 6 in [7].

Lemma 9 (lumped mass system, $\theta=1$ ). Under Assumptions A, $B, C, D, E, F$ and $H$,

$$
\left\{\begin{array}{l}
\left|\frac{n_{1} a_{n_{1}-1}((k+1) \Delta t)}{s_{n}((k+1) \Delta t)}-\frac{n_{1} a_{n_{1}-1}(k \Delta t)}{s_{n}(k \Delta t)}\right| \leqq M_{1} \Delta t^{1 / 2}, \\
\left|\frac{n_{2} a_{n_{1}+1}((k+1) \Delta t)}{L-s_{n}((k+1) \Delta t)}-\frac{n_{2} a_{n_{1}+1}(k \Delta t)}{L-s_{n}(k \Delta t)}\right| \leqq M_{2} \Delta t^{1 / 2}
\end{array}\right.
$$

Now we define a piecewise constant function

$$
\begin{gathered}
z_{n}(t)=\frac{\kappa_{1} n_{1} a_{n_{1}-1}(k \Delta t)}{s_{n}(k \Delta t)}+\frac{\kappa_{2} n_{2} a_{n_{1}+1}(k \Delta t)}{L-s_{n}(k \Delta t)}, \\
k \Delta t \leqq t<(k+1) \Delta t .
\end{gathered}
$$

Then from (4.5) we have

$$
s_{n}(t)=\int_{0}^{t} z_{n}(\tau) d \tau
$$


From Lemma 8, on the other hand,

$$
\frac{\partial \widetilde{u}_{1}}{\partial x}\left(s_{n}(t), t\right) \text { and } \quad \frac{\partial \widetilde{u}_{2}}{\partial x}\left(s_{n}(t), t\right)
$$

exist, and are given explicitly as

$$
\left\{\begin{array}{c}
-\kappa_{1} \frac{\partial \widetilde{u}_{1}}{\partial x}\left(s_{n}(t), t\right) \\
\quad=\kappa_{1} n_{1} \frac{a_{n_{1}-1}(k \Delta t)+\alpha\left\{a_{n_{1}-1}((k+1) \Delta t)-a_{n_{1}-1}(k \Delta t)\right\}}{s_{n}(k \Delta t)+\alpha\left\{s_{n}((k+1) \Delta t)-s_{n}(k \Delta t)\right\}} \\
\kappa_{2} \frac{\partial \widetilde{u}_{2}}{\partial x}\left(s_{n}(t), t\right) \\
\quad=\kappa_{2} n_{2} \frac{a_{n_{1}+1}(k \Delta t)+\alpha\left\{a_{n_{1}+1}((k+1) \Delta t)-a_{n_{1}+1}(k \Delta t)\right\}}{\left(L-s_{n}(k \Delta t)\right)-\alpha\left\{s_{n}((k+1) \Delta t)-s_{n}(k \Delta t)\right\}}
\end{array} .\right.
$$

Define

$$
\zeta_{n}(t)=-\kappa_{1} \frac{\partial \widetilde{u}_{1}}{\partial x}\left(s_{n}(t), t\right)+\kappa_{2} \frac{\partial \widetilde{u}_{2}}{\partial x}\left(s_{n}(t), t\right)
$$

then we have

Lemma 10 (lumped mass system, $\theta=1$ ).

$$
\left|z_{n}(t)-\zeta_{n}(t)\right| \leqq M_{3} \Delta t^{1 / 2}
$$

Proof. From $b_{m} \leqq s_{n}(t) \leqq b_{M}$ and Lemma 9, we have in $D_{1}$

$$
\begin{aligned}
n_{1}\left|\frac{a_{n_{1}-1}(k \Delta t)+\alpha\left\{a_{n_{1}-1}((k+1) \Delta t)-a_{n_{1}-1}(k \Delta t)\right\}}{s_{n}(k \Delta t)+\alpha\left\{s_{n}((k+1) \Delta t)-s_{n}(k \Delta t)\right\}}-\frac{a_{n_{1}-1}(k \Delta t)}{s_{n}(k \Delta t)}\right| \\
=\frac{\alpha s_{n}((k+1) \Delta t)}{s_{n}(k \Delta t)+\alpha\left\{s_{n}((k+1) \Delta t)-s_{n}(k \Delta t)\right\}} \\
\quad \times\left|\frac{n_{1} a_{n_{1}-1}((k+1) \Delta t)}{s_{n}((k+1) \Delta t)}-\frac{n_{1} a_{n_{1}-1}(k \Delta t)}{s_{n}(k \Delta t)}\right| \leqq \frac{\alpha b_{M}}{b_{m}} M_{1} \Delta t^{1 / 2} .
\end{aligned}
$$

We can verify a similar inequality in $D_{2}$, and from these inequalities in view of (4.12) and (4.15) we conclude (4.16). Q.E.D. 


$$
s_{n}(t)=\int_{0}^{t} \zeta_{n}(\tau) d \tau+\int_{0}^{t}\left(z_{n}(\tau)-\zeta_{n}(\tau)\right) d \tau
$$

As $\Delta t$ tends to zero the second term of the right hand side vanishes according to Lemma 10 , so that we have

$$
s_{\infty}(t)=\int_{0}^{t}\left\{-\kappa_{1} \frac{\partial \hat{u}_{1}}{\partial x}\left(s_{\infty}(\tau), \tau\right)+\kappa_{2} \frac{\partial \hat{u}_{2}}{\partial x}\left(s_{\infty}(\tau), \tau\right)\right\} d \tau,
$$

i.e.

$$
\frac{d s_{\infty}(t)}{d t}=-\kappa_{1} \frac{\partial \hat{u}_{1}}{\partial x}\left(s_{\infty}(t), t\right)+\kappa_{2} \frac{\partial \hat{u}_{2}}{\partial x}\left(s_{\infty}(t), t\right)
$$

This shows that $\widehat{u}_{1}, \widehat{u}_{2}$ and $s_{\infty}(t)$ satisfy (1.5).

Finally, the assumptions for the Stefan data made by Cannon and Primicerio [2] cover the assumptions in the present paper, and hence the solution of $(1.1)-(1.5)$ is unique [2], so that we have the main convergence

Theorem 2 (lumped mass system, $\theta=1$ ). Under Assumptions A, $B, C, D, E, F, G$ and $H$, the approximate solution obtained by (2.17)(2.21) converges to the solution of the Stefan problem (1.1)-(1.5) as $\Delta t \rightarrow 0, n_{1}, n_{2} \rightarrow \infty$.

This theorem also establishes the existence of the solution of (1.1)(1.5) under Assumptions A, B, C, D and H.

\section{§5. Improved Scheme}

Although the scheme given at the end of $\S 2$ is very simple and easy to compute, the speed of convergence has been observed to be a little slow. However, it can be remarkably improved with a slight modification of the scheme. The idea is to revise $\Delta s_{n}$ and $s_{n}$ at each step immediately after the new data are obtained. The improved scheme is as follows.

\section{Initial Routine:}




$$
\left\{\begin{aligned}
a_{j}(0)=f_{1}\left(x_{j}\right) ; j & =0,1, \cdots, n_{1}, \\
a_{j}(0)=f_{2}\left(x_{j}\right) ; j & =n_{1}+1, \cdots, n_{1}+n_{2}, \\
s_{n}(0) & =b
\end{aligned}\right.
$$

\section{General Routine:}

Repeat the following process for $k=1,2, \cdots, m$.

(i) Compute $\Delta s_{n}((k-1 / 2) \Delta t)$ and $s_{n}((k-1 / 2) \Delta t)$ using $\boldsymbol{a}_{\nu}((k-1) \Delta t)$ and $s_{n}((k-1) \Delta t)$ by means of

$$
\begin{aligned}
& \Delta s_{n}\left(\left(k-\frac{1}{2}\right) \Delta t\right) \\
& \quad=\left\{\frac{\kappa_{1} n_{1} a_{n_{1}-1}((k-1) \Delta t)}{s_{n}((k-1) \Delta t)}+\frac{\kappa_{2} n_{2} a_{n_{1}+1}((k-1) \Delta t)}{L-s_{n}((k-1) \Delta t)}\right\} \Delta t \\
& s_{n}\left(\left(k-\frac{1}{2}\right) \Delta t\right)=s_{n}((k-1) \Delta t)+\frac{1}{2} \Delta s_{n}\left(\left(k-\frac{1}{2}\right) \Delta t\right) .
\end{aligned}
$$

(ii) Compute $M_{\nu}, K_{\nu}, N_{\nu}, \nu=1,2$ using $\Delta s_{n}\left(\left(k-\frac{1}{2}\right) \Delta t\right)$ and $s_{n}\left(\left(k-\frac{1}{2}\right) \Delta t\right)$.

(iii) Solve the following linear equations for $\boldsymbol{a}_{1}(k \Delta t)$ and $\boldsymbol{a}_{2}(k \Delta t)$ :

$$
\begin{aligned}
\left\{M_{\nu}\right. & \left.+\theta \Delta t\left(\sigma_{\nu} K_{\nu}+N_{\nu}\right)\right\} \boldsymbol{a}_{\nu}(k \Delta t) \\
& =\left\{M_{\nu}-(1-\theta) \Delta t\left(\sigma_{\nu} K_{\nu}+N_{\nu}\right)\right\} \boldsymbol{a}_{\nu}((k-1) \Delta t), \nu=1,2 .
\end{aligned}
$$

(iv) Compute $\Delta s_{n}(k \Delta t)$ and $s_{n}(k \Delta t)$ using $\boldsymbol{a}_{\nu}(k \Delta t)$ and $s_{n}\left(\left(k-\frac{1}{2}\right) \Delta t\right)$ by means of

$$
\begin{gathered}
\Delta s_{n}(k \Delta t)=\left\{\frac{\kappa_{1} n_{1} a_{n_{1}-1}(k \Delta t)}{s_{n}\left(\left(k-\frac{1}{2}\right) \Delta t\right)}+\frac{\kappa_{2} n_{2} a_{n_{1}+1}(k \Delta t)}{L-s_{n}\left(\left(k-\frac{1}{2}\right) \Delta t\right)}\right\} \Delta t, \\
s_{n}(k \Delta t)=s_{n}\left(\left(k-\frac{1}{2}\right) \Delta t\right)+\frac{1}{2} \Delta s_{n}(k \Delta t) .
\end{gathered}
$$

We can show in almost the same way as in that of $\S 3$ that Theorem 1 also holds for the stability of the improved scheme under Assumption $\mathrm{A}, \mathrm{B}, \mathrm{C}, \mathrm{D}, \mathrm{E}$ and $\mathrm{F}$. In the present case the arguments $k \Delta t$ of $\lambda_{1}, \lambda_{2}, \beta_{1}, \beta_{2}$ in (3.3) and (3.4) should be replaced by $\left(k-\frac{1}{2}\right) \Delta t$. Then it is easy to see that we have

$$
-\gamma_{-}\left(1-\frac{1}{2 n_{2}}\right) \leqq \frac{\Delta s_{n}\left(\left(l-\frac{1}{2}\right) \Delta t\right)}{\Delta t} \leqq \gamma_{+}\left(1-\frac{1}{2 n_{1}}\right)
$$

in place of (3.14), and 


$$
b_{m} \leqq s_{n}\left(\left(l-\frac{1}{2}\right) \Delta t\right) \leqq b_{M}
$$

in place of (3.19). In the proof of Lemma 3 we must derive $s_{n}\left(\left(l-\frac{1}{2}\right) \Delta t\right) \leqq b_{M}$ from $s_{n}((l-1) \Delta t) \leqq b_{M}-\delta, 0 \leqq \delta \leqq \frac{1}{2} \gamma_{+} \Delta t$ in stead of (3.21). For this purpose we need $\frac{1}{2} F_{+}(\delta) \leqq \delta$ and $\frac{1}{2} F_{+}\left(\frac{1}{2} \gamma_{+} \Delta t\right)$ $\leqq \frac{1}{2} \gamma_{+} \Delta t$, which, however, follow from $F_{+}(\delta) \leqq \delta$ and $F_{+}\left(\gamma_{+} \Delta t\right) \leqq \gamma_{+} \Delta t$. Therefore at each step from $(k-1) \Delta t$ to $\left(k-\frac{1}{2}\right) \Delta t$ the scheme given by (3.3) and (3.4) is stable. Then we repeat the same reasoning once more at the step from $\left(k-\frac{1}{2}\right) \Delta t$ to $k \Delta t$ replacing $a_{n_{1}-1}^{l-1}$ by $a_{n_{1}-1}^{l}$ and $a_{n_{1}+1}^{l-1}$ by $a_{n_{1}+1}^{l}$ in (3.12) and also replacing $s_{n}((l-1) \Delta t)$ by $s_{n}\left(\left(l-\frac{1}{2}\right) \Delta t\right)$ in (3.13), so that we have (3.14) and (3.19). Hence we have

Theorem $\mathbb{1}^{\prime}$ (improved scheme). Under Assumptions $A, B, C, D$, $E$ and $F$ the scheme (5.1)-(5.7) is stable in the sense that the maximum principle (3.50) holds.

In order to prove the convergence of the improved scheme, we extend $s_{n}(k \Delta t)$ defined at discrete points to a continuous function in the following way:

$$
\begin{gathered}
s_{n}(t)=\left\{\begin{array}{l}
s_{n}(k \Delta t)+\alpha \Delta s_{n}\left(\left(k+\frac{1}{2}\right) \Delta t\right) ; k \Delta t<t \leqq\left(k+\frac{1}{2}\right) \Delta t, \\
s_{n}\left(\left(k+\frac{1}{2}\right) \Delta t\right)+\left(\alpha-\frac{1}{2}\right) \Delta s_{n}(k \Delta t) ;\left(k+\frac{1}{2}\right) \Delta t<t \leqq k \Delta t,
\end{array}\right. \\
\alpha=\frac{t-k \Delta t}{\Delta t} .
\end{gathered}
$$

The extention of $a_{j}(t)$, i.e. that of $\widetilde{u}_{1}$ and $\widetilde{u}_{2}$, to intermediate values of $t$ is exactly the same as is done in $\S 4$. Then we can again extract a subsequence from $\left\{s_{n}(t)\right\}$ that converges.

In the definition of $c_{j}^{k}$ of $(4.9)$ we replace $h_{\nu}((k-1) \Delta t)$ by $h_{\nu}\left(\left(k-\frac{1}{2}\right) \Delta t\right)$.

$z_{n}(t)$ in Lemma 10 should be modified to be 
(5. 11)

$$
z_{n}(t)=\left\{\begin{array}{c}
\frac{\kappa_{1} n_{1} a_{n_{1}-1}(k \Delta t)}{s_{n}(k \Delta t)}+\frac{\kappa_{2} n_{2} a_{n_{1}+1}(k \Delta t)}{L-s_{n}(k \Delta t)} ; \\
k \Delta t<t \leqq\left(k+\frac{1}{2}\right) \Delta t \\
\frac{\kappa_{1} n_{1} a_{n_{1}-1}((k+1) \Delta t)}{s_{n}\left(\left(k+\frac{1}{2}\right) \Delta t\right)}+\frac{\kappa_{2} n_{2} a_{n_{1}+1}((k+1) \Delta t)}{L-s_{n}\left(\left(k+\frac{1}{2}\right) \Delta t\right)} \\
\left(k+\frac{1}{2}\right) \Delta t<t \leqq(k+1) \Delta t
\end{array}\right.
$$

In regard to the extended solution $\tilde{u}_{1}$ we have

$$
\begin{aligned}
& -\kappa_{1} \frac{\partial \widehat{u}_{1}}{\partial x}\left(s_{n}(t), t\right) \\
& =\left\{\begin{array}{c}
\kappa_{1} n_{1} a_{n_{1}-1}(k \Delta t)+\alpha\left\{a_{n_{1}-1}((k+1) \Delta t)-a_{n_{1}-1}(k \Delta t)\right\} \\
s_{n}(k \Delta t)+2 \alpha\left\{s_{n}\left(\left(k+\frac{1}{2}\right) \Delta t\right)-s_{n}(k \Delta t)\right\} \\
k \Delta t<t \leqq\left(k+\frac{1}{2}\right) \Delta t \\
\kappa_{1} n_{1} \frac{a_{n_{1}-1}(k \Delta t)+\alpha\left\{a_{n_{1}-1}((k+1) \Delta t)-a_{n_{1}-1}(k \Delta t)\right\}}{s_{n}\left(\left(k+\frac{1}{2}\right) \Delta t\right)+(2 \alpha-1)\left\{s_{n}((k+1) \Delta t)-s_{n}\left(\left(k+\frac{1}{2}\right) \Delta t\right)\right\}} \\
\left(k+\frac{1}{2}\right) \Delta t<t \leqq(k+1) \Delta t
\end{array}\right.
\end{aligned}
$$

and hence the estimates we need for the proof of Lemma 10 become

$$
\left\{\begin{array}{c}
n_{1} \mid \frac{a_{n_{1}-1}(k \Delta t)+\alpha\left\{a_{n_{1}-1}((k+1) \Delta t)-a_{n_{1}-1}(k \Delta t)\right\}}{s_{n}(k \Delta t)+2 \alpha\left\{s_{n}\left(\left(k+\frac{1}{2}\right) \Delta t\right)-s_{n}(k \Delta t)\right\}} \\
-\frac{a_{n_{1}-1}(k \Delta t)}{s_{n}(k \Delta t)} \mid \leqq M_{1}^{\prime} \Delta t^{1 / 2}, \\
n_{1} \mid \begin{array}{c}
a_{n_{1}-1}(k \Delta t)+\alpha\left\{a_{n_{1}-1}((k+1) \Delta t)-a_{n_{1}-1}(k \Delta t)\right\} \\
s_{n}\left(\left(k+\frac{1}{2}\right) \Delta t\right)+(2 \alpha-1)\left\{s_{n}((k+1) \Delta t)-s_{n}\left(\left(k+\frac{1}{2}\right) \Delta t\right)\right\} \\
-\frac{a_{n_{1}-1}((k+1) \Delta t)}{s_{n}\left(\left(k+\frac{1}{2}\right) \Delta t\right)} \mid \leqq M_{1}^{\prime \prime} \Delta t^{1 / 2},
\end{array}
\end{array}\right.
$$

which are shown to be valid in a similar way as that in $\S 4$ using (4.11) in view of $\left|\Delta s_{n}\left(\left(k+\frac{1}{2}\right) \Delta t\right)\right| \leqq \gamma \Delta t$ and $\left|\Delta s_{n}((k+1) \Delta t)\right| \leqq \gamma \Delta t$. The situation is the same for $\widetilde{u}_{2}$, so that for the convergence of the improved scheme we have

Theorem 2' (improved scheme, lumped mass system, $\theta=1$ ). Under 
Assumptions $A, B, C, D, E, F, G$ and $H$, the approximate solution obtained by (5.1)-(5.7) converges to the solution of the Stefan problem (1.1)-(1.5) as $\Delta t \rightarrow 0, n_{1}, n_{2} \rightarrow \infty$.

\section{§6. Numerical Example}

We applied the present method to the following problem:

$$
0 \leqq x \leqq L=2,
$$

$$
\sigma_{1}=5, \quad \sigma_{2}=10, \quad \kappa_{1}=\frac{1}{10}, \quad \kappa_{2}=\frac{3}{10},
$$

$$
\left\{\begin{array}{l}
g_{1}(t)=1 \\
g_{2}(t)= \begin{cases}-\frac{3}{8}\left(\cos \pi t+\frac{5}{3}\right) & ; 0 \leqq t \leqq 1 \\
-\frac{1}{4} & ; 1<t \leqq 5\end{cases}
\end{array}\right.
$$

$$
\begin{gathered}
s(0)=b=1, \\
\left\{\begin{array}{l}
f_{1}(x)=1-x, \\
f_{2}(x)=1-x
\end{array}\right.
\end{gathered}
$$

The computation was carried out by means of the improved scheme of the lumped mass system with $\theta=1$. We employed two sets of parameters :

$$
\unlhd t=0.1 . \quad n_{1}=n_{2}=5 \text {. }
$$

$$
\Delta t=0.001, \quad n_{1}=n_{2}=10 \text {. }
$$

The data and the parameters satisfy all the assumptions we have made, and the computation was actually stable. Fig. 3 shows the change of $s_{n}(t)$ obtained using the parameters (6.6). It can be shown theoretically that $s(t)$ approaches to $8 / 7$ asymptotically in the present case. It turned out that, even with the coarser mesh sizes (6.6), the accuracy of the result was good enough. In fact, the differences of $\widetilde{u}_{1}, \widetilde{u}_{2}$ and 


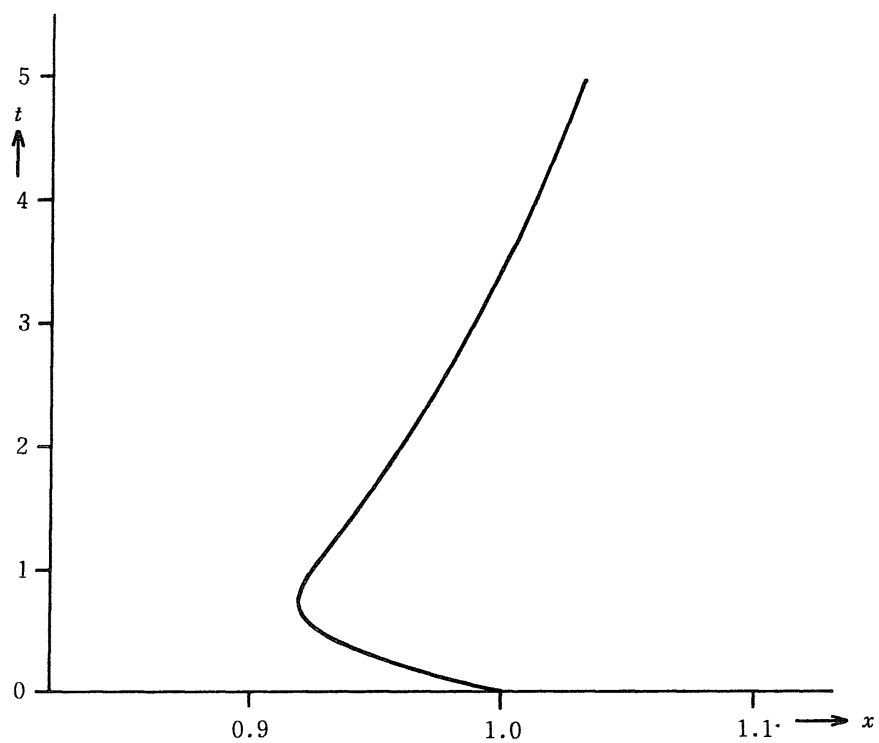

Fig. 3. The change of $s_{n}(t)$.

$s_{n}(t)$ with (6.6) and those with the finer parameters (6.7) are less than $10^{-3}$ at the points corresponding to the mesh points of the solution obtained with (6.6).

\section{References}

[1] Bonnerot, R. and Jamet, P., A second order finite element method for the onedimensional Stefan problem, Internat. J. Numer. Methods Engrg., 8 (1974), 811820.

[2] Cannon, J. R. and Primicerio, M., A two phase Stefan problem with temperature boundary conditions, Ann. Mat. Pura Appl. (IV) 88 (1971), 177-191.

[3] - A two phase Stefan problem with flux boundary conditions, Ann. Mat. Pura Appl. (IV) 88 (1971), 193-205.

[4] Douglas, J. Jr. and Gallie, T. M., On the numerical integration of a parabolic differential equation subject to a moving boundary condition, Duke Math. J., 22 (1955), 557-571.

[5] Kawarada, H. and Natori, M., On numerical solutions of Stefan problem I, Mem. Numer. Math., No. 1 (1975), 43-54.

[6] Meyer, G. H., On a free interface problem for linear ordinary differential equations and the one phase Stefan problem, Numer. Math., 16 (1970), 248-267.

[7] Mori, M., Stability and convergence of a finite element method for solving the Stefan problem, Publ. RIMS, Kyoto Univ., 12 (1976), 539-563.

[8] - A finite element method for solving moving boundary problems, Preprints of IFIP Working Conference on Modelling of Environmental Systems, Tokyo, April, 1976, 167-171. 
[9] Stability of a finite element method for solving the Stefan problem in one space dimension, Proceedings of the Japan-France Seminar on Numerical Analysis and Functional Analysis, Tokyo and Kyoto, September, 1976.

[10] Nogi, T., A difference scheme for solving the Stefan problem, Publ. RIMS, Kyoto Univ., 9 (1974), 543-575. 
